\title{
CINÉTICA DE LA HIDROGENACIÓN CATALÍTICA SELECTIVA DE 1-BUTINO Y 1,3-BUTADIENO EN PRESENCIA DE N-BUTENOS.
}

\author{
Alves, Javier A. ${ }^{a, b *}$, Bressa Sergio P. ${ }^{a, b}$, Martínez Osvaldo M. ${ }^{a, b}$, Barreto Guillermo F. ${ }^{a, b}$ \\ ${ }^{a}$ PROIRQ, Área Departamental Ingeniería Química, Facultad de Ingeniería (UNLP), La Plata, CP1900 \\ - Argentina

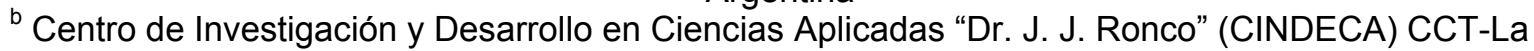 \\ Plata-CONICET- UNLP, calle 47 No. 257, CP 1900, La Plata, Argentina \\ *54-221-4210711(Int. 125) - jalves@quimica.unlp.edu.ar
}

Palabras claves: Hidrogenación selectiva, 1-3 butadieno, 1-butino, 1-buteno, cinética

\section{INTRODUCCIÓN.}

La purificación de cortes ricos en $\mathrm{C}_{4}$ `s, para la obtención industrial de 1-buteno (1BE) de alta pureza, se realiza mediante la hidrogenación selectiva de 1,3 - butadieno (BD) y compuestos acetilénicos sobre catalizadores de $\mathrm{Pd} / \mathrm{Al}_{2} \mathrm{O}_{3}$ hasta alcanzar los requerimientos especificados para el producto [1]. El 1BE de alta pureza se emplea en la producción de polibuteno y polietileno lineal de baja densidad. La remoción de las impurezas no es económicamente factible mediante una destilación debido a la cercanía de los puntos de ebullición de los hidrocarburos involucrados.

Las tecnologías corrientes utilizan un reactor catalítico de lecho fijo con la mezcla de hidrocarburos e $\mathrm{H}_{2}$ fluyendo en co-corriente en flujo ascendente o descendente [1]. Las temperaturas de operación van desde valores ambientes hasta alrededor de $50-60{ }^{\circ} \mathrm{C}$. Las presiones son lo suficientemente altas como para mantener los hidrocarburos en fase líquida. Concentraciones típicas para el BD y el 1-butino (BY) en estos cortes son de alrededor del $1 \%$ molar. Luego de la purificación catalítica de esta corriente el contenido de diolefinas y compuestos acetilénicos no debe superar las 10 ppm. Estas especificaciones deben lograrse con mínimas pérdidas de $1 \mathrm{BE}$.

Los procesos de hidrorefinado, destinados a la hidrogenación selectiva, son llevados a cabo empleando catalizadores que aporten el requisito deseado de selectividad. Es reconocido en la literatura que, entre los elementos del Grupo VIII, el Pd es el agente más selectivo para la hidrogenación de compuestos acetilénicos y diolefinas [2,3]. De esta forma, pequeñas cantidades de BY y BD serán suficientes para inhibir la adsorción y reacción de

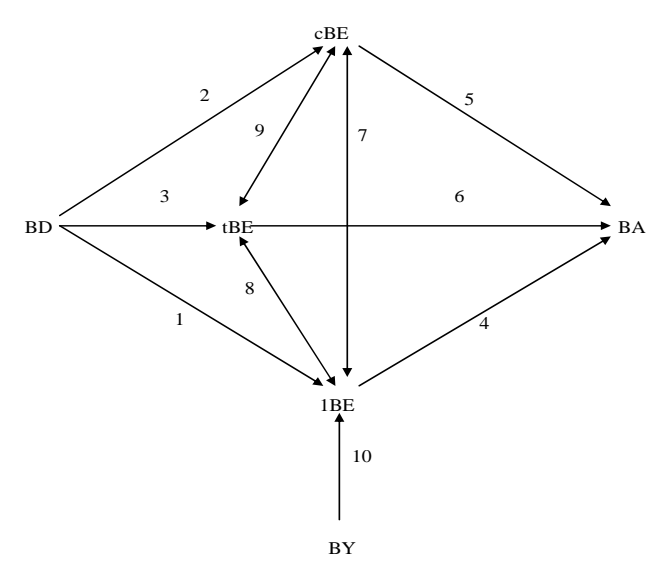

Figura 1: Esquema global de reacciones. Si bien en la bibliografía existen numerosos
estudios sobre hidrogenación selectiva de
compuestos insaturados, la mayoría se centraron en los efectos del tamaño de partícula del
metal, la distribución de productos, intermediarios de reacción, mecanismos catalíticos, y la los n-butenos. Otra característica sobresaliente del $\mathrm{Pd}$ es su elevada actividad para la hidrogenación de hidrocarburos insaturados y para la hidro-isomerización de olefinas. Este comportamiento del $\mathrm{Pd}$ ofrece ventajas $\mathrm{y}$ desventajas para los procesos de hidrorefinado. Una velocidad de hidrogenación elevada de compuestos acetilénicos y BD es ventajosa, pues permite la purificación de corrientes de nbutenos a bajas temperaturas. La facilidad de hidrogenación e hidro-isomerización del $1 \mathrm{BE}$ es perjudicial por cuanto conduce a pérdidas importantes de la sustancia valiosa. El conjunto de reacciones considerado se representa en la Figura 1. 
influencia de los oligómeros, promotores y aditivos sobre la actividad y selectividad de los catalizadores [4].

Tal como fuera analizado en [5], son escasos los estudios en los cuales se reportan expresiones cinéticas y valores de los parámetros de las mismas que sean aplicables al estudio del comportamiento del reactor industrial.

En [6] se empleó un catalizador de tipo egg-shell de $\mathrm{Pd} / \mathrm{Al}_{2} \mathrm{O}_{3}$, realizando estudios en fase gaseosa y líquida. Para distintas composiciones iniciales de mezclas de BY y BD se analizó la dependencia de la velocidad de reacción con la concentración de hidrocarburos e hidrógeno, pero no se reportaron valores de los parámetros cinéticos.

En [7] trabajó en condiciones de presión y temperatura similares a las industriales, se realizó un análisis de la forma de las expresiones cinéticas, se reportaron valores de energías de activación, se presentaron solamente relaciones de los parámetros cinéticos pero sus valores individuales no fueron informados.

En [5] se realizó un estudio cinético de este sistema reactivo en fase líquida a una única temperatura, sobre un catalizador comercial del tipo cáscara de huevo. Se llevó adelante un análisis de discriminación entre dos modelos cinéticos propuestos, uno derivado de suponer la adsorción de una molécula de BD sobre un único sitio activo y el otro sobre dos sitios activos.

En [8] se publicó un estudio experimental en fase líquida de la hidrogenación de BY. Estos resultados se analizan solo hasta concentraciones de BY superiores a $1000 \mathrm{ppm}$. El análisis se extendió hasta 20 ppm de BY en [9], que es el nivel requerido en los reactores industriales.

En este contexto, el objetivo del presente trabajo es ajustar parámetros cinéticos que no habían podido ser evaluados anteriormente, específicamente energías de activación y una constante de adsorción del BY. Con este fin, se amplía el rango de condiciones de trabajo, a través de ensayos realizados a distintos niveles de temperatura y de mezclas reactivas en las que están presentes desde el inicio de la reacción el BY y el BD, no analizados previamente.

\section{EXPERIMENTAL}

\subsection{Procedimiento del trabajo experimental.}

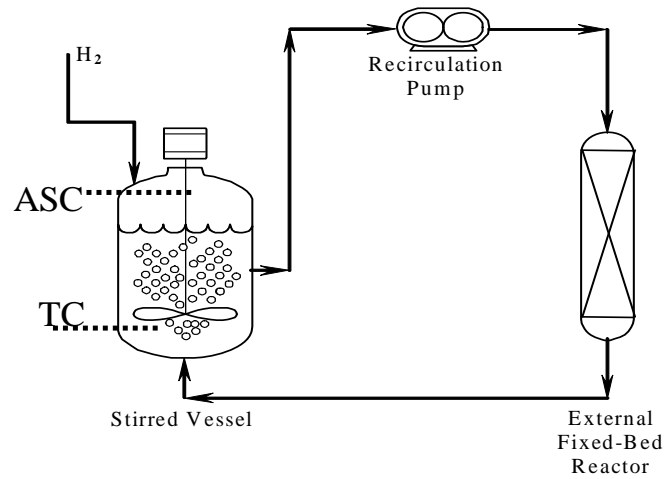

Figura 2: esquema del equipo experimental

El catalizador comercial contiene Pd al 0,2\% w / w sobre alúmina, en esferas de $2,34 \mathrm{~mm}$ de diámetro, con un espesor de capa activa de 230 micras.

Un vaso agitado de $100 \mathrm{ml}$, con una turbina impulsada mecánicamente de capacidad, aloja el lote de la mezcla reactiva de hidrocarburos y se encuentra conectado a una línea de suministro continuo de $\mathrm{H}_{2}$. En la Figura 2 se presenta un esquema simplificado del equipo experimental.

Las reacciones se llevan a cabo en un lecho fijo externo al tanque agitado, donde se encuentra alojado el catalizador. Las partículas catalíticas se encuentran en su tamaño original. Una bomba permite la recirculación de la mezcla reactiva entre el tanque y el lecho externo. Se emplea como solvente líquido el n-hexano. 
Durante el desarrollo de los ensayos se extraen muestras de la fase líquida que son analizadas cromatógráficamente, obteniéndose valores de composición de la fase líquida para cada tiempo de muestreo. Detalles adicionales del sistema experimental se describen en [10].

\section{RESULTADOS EXPERIMENTALES}

El comportamiento del sistema se explica a partir de reconocer el efecto de fuertes resistencias difusionales dentro de la capa activa de las partículas del catalizador, un hecho que fue verificado con anterioridad ([5], [11], [12]).

\section{Influencia de la temperatura.}

A los fines de estudiar el efecto de la temperatura sobre el sistema de reacción, se realizó un conjunto de tres ensayos, en cada uno de los cuales estaba presente un hidrocarburo insaturado, BD, $1 \mathrm{BE}$ o cBE, en alta concentración (alrededor del 10\%) y dos o tres niveles térmicos entre 25 y $65^{\circ} \mathrm{C}$.

Como fuera detallado en [5], los ensayos presentan distintas actividades debido a la desactivación del catalizador. En ensayos realizados a diferentes niveles de temperatura, este fenómeno enmascara la incidencia de ésta sobre la velocidad de reacción observada.

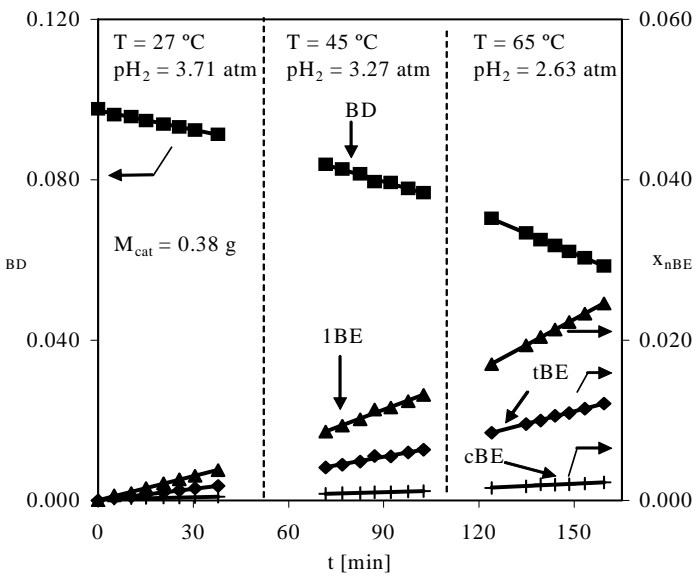

Figura. 3 Valores experimentales y predichos por el modelo.

Para evitar este efecto, se diseñaron experiencias en las que se modificó la temperatura durante el desarrollo de cada ensayo. De este modo las energías de activación de los coeficientes cinéticos pueden evaluarse independientemente de las actividades propias de cada ensayo. Para ejemplificar esta metodología, se muestra el ensayo de la Figura 3. Se trabaja con una alta concentración de un hidrocarburo, BD al 10\%, de manera que a lo largo del ensayo éste se encuentra inhibiendo las reacciones de las olefinas, tal como fuera verificado anteriormente ([5], [11]). Se generaron zonas de interés, cada una con temperatura específica, las cuales se encuentran delimitadas por rectas verticales. Inicialmente se trabaja a $27^{\circ} \mathrm{C}$, una vez extraídas un número considerado suficiente de muestras, se aumenta la temperatura a $45^{\circ} \mathrm{C}$. Cuando el sistema alcanza el nuevo estado estacionario, se extraen las muestras que corresponden a la temperatura de $45^{\circ} \mathrm{C}$. Por último, se aumenta la temperatura a $65^{\circ} \mathrm{C}$, y se extraen muestras en el estado estacionario.

\section{Selectividad del catalizador para hidrogenar BD y BY}

A fin de evaluar la selectividad del catalizador se realizaron dos ensayos en los que se encuentran presentes conjuntamente desde el inicio de la reacción, el BD y el BY. Se ha verificado previamente ([11], [5]), en ensayos en los que el BD se encuentra solo saturando la superficie catalítica, que su reacción de hidrogenación es la única que se produce. En estas circunstancias el BD se consume observándose un orden cero de reacción. En ensayos en donde es el BY quien se encuentra saturando la superficie catalítica [8], su reacción de hidrogenación es la única que se produce, verificándose un orden de reacción negativo.

En el ensayo de la Figura 4 aparecen el BD y el BY en una concentración inicial del $5.69 \%$ y $2 \%$ respectivamente. No se encuentran graficadas en las figuras las curvas 


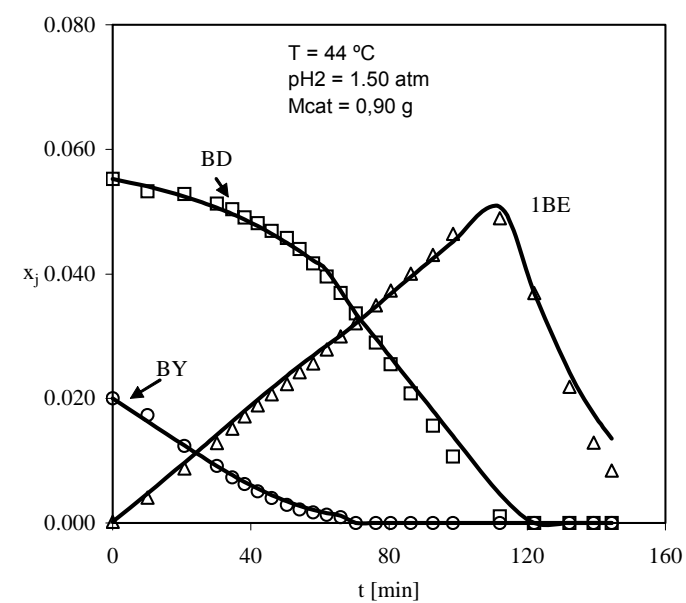

Figura. 4: Valores experimentales y predichos por el modelo.

correspondientes a los 2-butenos para facilitar la interpretación. Se aprecia que el BD y BY reaccionan simultáneamente, aunque el $B D$ se encuentra en mayor concentración, reacciona más lentamente al inicio, para luego acelerar su velocidad de reacción a medida que el $B Y$ se consume. Asimismo el BD compite con el BY cuando la concentración de éste disminuye lo suficiente, lo cual se puede comprobar comparando el comportamiento del BY en el ensayo de la Figura 4 con resultados previos ( Figura 2 en [8]) donde se encuentra solamente como reactivo insaturado inicial el BY. Allí se puede apreciar claramente el orden de reacción negativo del mismo. En cambio, en la Figura 4 el orden dejó de ser negativo antes por la presencia del BD. Por otra parte, el característico orden cero de reacción del $\mathrm{BD}$ ([11], [5]), presentado en ausencia del acetilénico, se ve postergado apareciendo a mayores tiempos, en los cuales el BY ha disminuido considerablemente su concentración. Este ensayo nos permite entonces determinar que el BD y el BY son competitivos para cierto nivel relativo de concentraciones. Luego, una vez extinguido el BY, y para un contenido de BD lo suficientemente bajos, aparecen las reacciones de las olefinas ([11], [5]).

\section{MODELO CINÉTICO.}

En [5] se propusieron dos modelos cinéticos, resultando que no se verificaron diferencias estadísticamente significativas entre ellos. Se adoptará el conjunto de expresiones cinéticas correspondientes al modelo I, derivado de suponer la adsorción de una molécula de $\mathrm{BD}$ sobre un único sitio activo, ya que permite una relativa mayor estabilidad durante la ejecución de los programas de regresión. En la Tabla II aparecen las etapas elementales del mecanismo catalítico.

Tabla II: mecanismo catalítico.

\begin{tabular}{|c|c|c|c|}
\hline Etapa & Mecanismo & & Mecanismo \\
\hline a) & $\mathrm{H}_{2}+2 \mathrm{e} f 2 \mathrm{H} \mathrm{e}$ & j) & $\mathrm{C}_{4} \mathrm{H}_{7}+\mathrm{He}{ }^{\circledR} 1 \mathrm{BE}+\mathrm{e}+{ }^{\prime}$ \\
\hline b) & $\mathrm{BD}+f^{\prime} \mathrm{BD}$ & l) & $\mathrm{C}_{4} \mathrm{H}_{7}+\mathrm{He}{ }^{\circledR}{ }^{\prime} \mathrm{tBE}+\mathrm{e}+{ }^{\prime}$ \\
\hline c) & $\mathrm{BY}+\times f \times \mathrm{BY}$ & m) & $\times\left(\mathrm{C}_{4} \mathrm{H}_{7}\right)^{\mathrm{BY}}+\mathrm{He}{ }^{\circledR} \times 1 \mathrm{BE}+\mathrm{e}$ \\
\hline d) & $\times \mathrm{BY}+\mathrm{BY} f \times(\mathrm{BY})_{2}$ & n) & $1 \mathrm{BE}+\mathrm{He} f{ }^{\prime} \mathrm{C}_{4} \mathrm{H}_{9}{ }^{1}+\mathrm{e}$ \\
\hline $\begin{array}{l}\text { e) } \\
\text { f) } \\
\text { g) }\end{array}$ & $\begin{array}{l}1 \mathrm{BE}+^{\prime} f^{\prime} 1 \mathrm{BE} \\
\mathrm{cBE}^{\prime} f^{\prime} \mathrm{cBE} \\
\mathrm{tBE}+^{\prime} f^{\prime} \mathrm{tBE}\end{array}$ & $\begin{array}{l}\text { o) } \\
\text { p) } \\
\text { q) }\end{array}$ & $\begin{array}{l}1 \mathrm{BE}+\mathrm{He} f^{\prime} \mathrm{C}_{4} \mathrm{H}_{9}{ }^{2}+\mathrm{e} \\
\mathrm{cBE}+\mathrm{He} f \\
\mathrm{fBE}+\mathrm{He} f\end{array}$ \\
\hline h) & $\mathrm{BD}+\mathrm{He} f^{\prime} \mathrm{C}_{4} \mathrm{H}_{7}+\mathrm{e}$ & r) & $\mathrm{C}_{4} \mathrm{H}_{9}{ }^{1}+\mathrm{He}{ }^{\circledR} \mathrm{BA}+{ }^{\prime}+\mathrm{e}$ \\
\hline i) & $\times \mathrm{BY}+\mathrm{He} f \times\left(\mathrm{C}_{4} \mathrm{H}_{7}\right)^{\mathrm{BY}}+\mathrm{e}$ & s) & $\mathrm{C}_{4} \mathrm{H}_{9}{ }^{2}+\mathrm{He} \stackrel{B}{ } \mathrm{BA}^{\prime}+\mathrm{e}$ \\
\hline
\end{tabular}

Para el $\mathrm{H}_{2}$ se consideró una adsorción disociativa, indicada como la etapa a) en la Tabla II, en sitios $(\odot)$ diferentes a los $(x)$ involucrados en la adsorción de las especies de hidrocarburos. El planteo de un mecanismo "no competitivo" es ampliamente aceptado en la literatura ([3], [13] y sus referencias). 
Las etapas de la Tabla II correspondientes a la adsorción del BD, etapa (b), así como las reacciones de hidrogenación de sus intermediarios superficiales, etapas (h), (j) - (I) fueron propuestos en [14].

Respecto a las etapas de adsorción del BY, se ha verificado que solo el propuesto en [15] y [16], permite alcanzar una expresión cinética que, bajo ciertas condiciones operativas, tiene un orden negativo para el BY. Se forman dos especies superficiales para el BY: una constituida por una única molécula de BY adsorbida sobre un sitio activo de metal ( $\pi-$ adsorbido), que es el intermediario activo, y la otra consistente en dos moléculas de BY y un sitio metálico ( $\sigma$-diadsorbido), formando un complejo estable no reactivo. Ambas especies se encuentran en equilibrio, de acuerdo a la etapa elemental (d) de la Tabla II.

El resto de las etapas elementales remanentes, n)-s), correspondientes a las especies superficiales de los n-butenos, son el resultado de experiencias previas en fase vapor [13]. Se realizaron las siguientes suposiciones durante la derivación:

1) Todas las etapas de adsorción, etapas a)-g) en la Tabla II, están equilibradas, es decir que sus velocidades son muy grandes comparadas con las de las etapas limitantes.

2) La cantidad de sitios " $\mathrm{x}$ " ocupados por los radicales semi-hidrogenados $\mathrm{xC}_{4} \mathrm{H}_{7}, \mathrm{x}\left(\mathrm{C}_{4} \mathrm{H}_{7}\right)^{\mathrm{BY}}$,", $\mathrm{xC}_{4} \mathrm{H}_{9}{ }^{1}$ y $\mathrm{xC}_{4} \mathrm{H}_{9}{ }^{2}$, resultan despreciables frente a la concentración total de sitios activos, [ $]_{1}$.

Dado que la adsorción de todas las sustancias participantes precede a sus reacciones, las expresiones cinéticas que se deriven resultarán del tipo Langmuir-Hinshelwood (LHHW).

Las expresiones de las velocidades de reacción, para el conjunto de reacciones globales definido en la Figura 1 , se dan en la Tabla III, donde $y_{i}$ representa un valor puntual de la fracción molar de la especie j en la fase líquida dentro de los poros de la capa activa.

Como se observa, las fuerzas impulsoras de todas las ecuaciones de las velocidades de reacción exhiben un orden uno con respecto a las fracciones molares de los hidrocarburos, en tanto que las velocidades de las reacciones de hidrogenación muestran un orden uno con respecto al $\mathrm{H}_{2}$, mientras que las correspondientes a las reacciones de hidroisomerización exhiben una dependencia a la raíz cuadrada de la fracción molar del $\mathrm{H}_{2}$. vínculos

No todos los parámetros son independientes, debiéndose considerar los siguientes $\mathrm{k}_{4}^{\mathrm{II}}=\frac{\mathrm{k}_{5} \mathrm{k}_{8}}{\mathrm{k}_{9}}(1) ; \quad \mathrm{k}_{6}=\frac{\mathrm{k}_{5} \mathrm{k}_{8}}{\mathrm{k}_{7} \mathrm{~K}_{\mathrm{r}, \mathrm{t}}^{\mathrm{eq}}}(2) ; \gamma=\frac{\mathrm{k}_{5}}{\mathrm{k}_{9}\left(1+\mathrm{k}_{7} / \mathrm{k}_{8}\right)+\mathrm{k}_{7} / \mathrm{K}_{1-\mathrm{c}}^{\mathrm{eq}}}(3)$. Las constantes de equilibrio
químico son evaluadas termodinamicamente a cada temperatura de trabajo.

\section{REGRESIÓN DE LOS DATOS EXPERIMENTALES}

El conjunto de subrutinas empleado para realizar la regresión de los datos experimentales puede dividirse en dos bloques. El bloque GREGPAK [17], permite obtener las estimaciones óptimas de los parámetros cinéticos a través de la comparación de los valores de fracciones molares en el seno de la fase líquida predichos por el modelo y experimentales. Por otra parte, dadas las severas limitaciones difusionales que presenta el catalizador, el bloque VEL resuelve mediante un algoritmo [18], las ecuaciones de conservación dentro de la capa activa. De esta forma se obtienen valores de velocidades de reacción observadas las cuales son requeridas por el bloque GREGPAK. Detalles sobre la formulación y la solución numérica se dan en $[5,10,12]$.

Respecto de los términos de inhibición del $\mathrm{H}_{2}$, en [12] se hicieron ensayos de sensibilidad con diferentes juegos de valores de los parámetros $\alpha, \beta, \mathrm{K}_{\mathrm{H} 2}$, concluyéndose

Tabla III: expresiones cinéticas derivadas del mecanismo. 


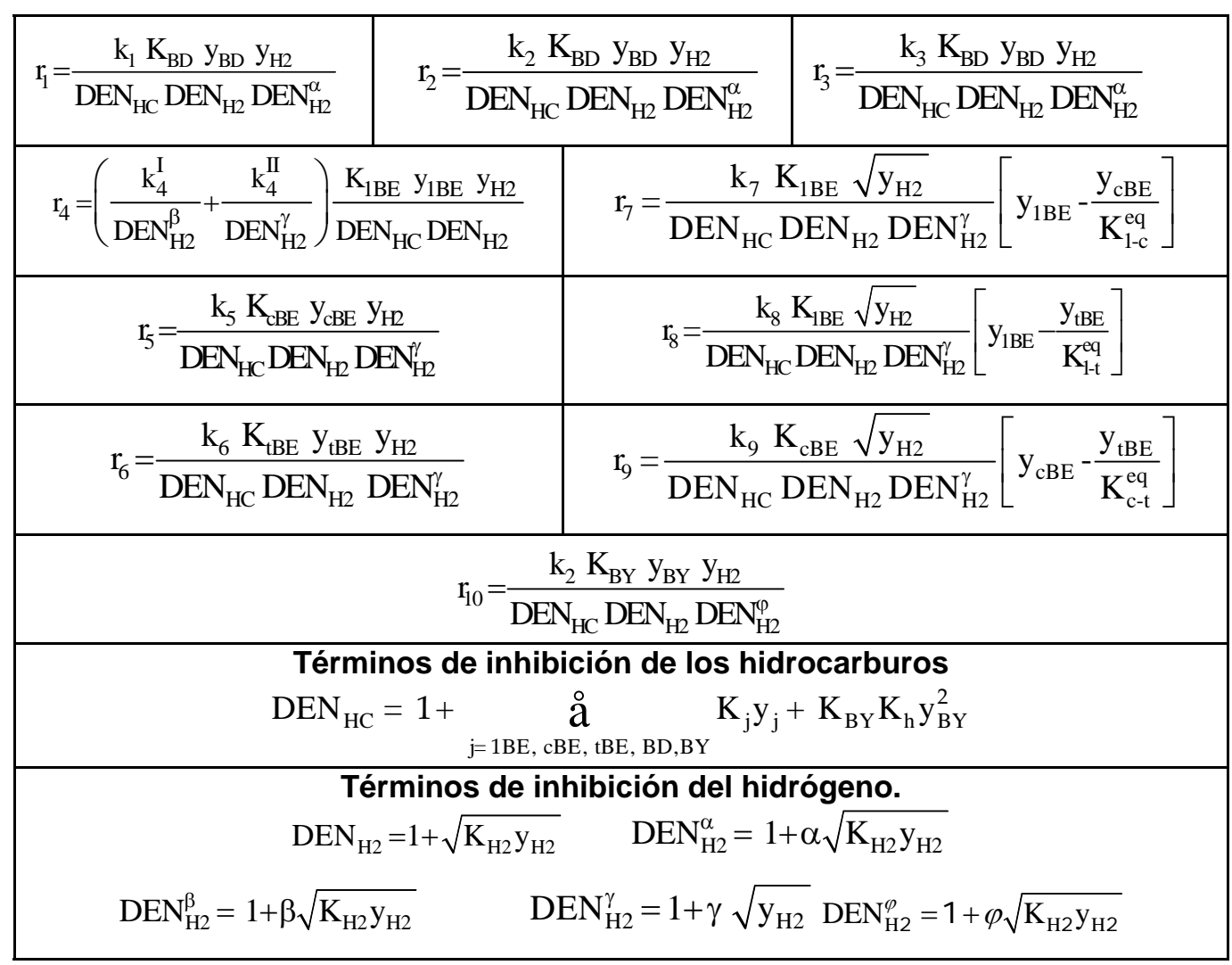

que la mejor opción resulta fijar durante la regresión los términos $\mathrm{DEN}_{\mathrm{H} 2}=\mathrm{DEN}_{\mathrm{H} 2}^{\alpha}$ $=\mathrm{DEN}_{\mathrm{H} 2}^{\beta}=\mathrm{DEN}_{\mathrm{H} 2}^{\varphi}=1$.

La funcionalidad de los coeficientes cinéticos con la temperatura se expresó de la siguiente manera:

$$
\mathrm{k}(\mathrm{T})=\mathrm{k}\left(\mathrm{T}_{\text {ref }}\right) \exp \left[-\frac{\mathrm{E}_{\mathrm{a}}}{\mathrm{R}}\left(\frac{1}{\mathrm{~T}}-\frac{1}{\mathrm{~T}_{\text {ref }}}\right)\right]
$$

en las cuales $\mathrm{E}_{\mathrm{a}}$ simboliza la energía de activación, $\mathrm{T}$ indica el nivel de temperatura, y $\mathrm{R}$ es la constante universal del estado gaseoso. En los ensayos, $\mathrm{k}\left(\mathrm{T}_{\mathrm{ref}}\right)$ es el coeficiente cinético propio de cada ensayo que corresponde al intervalo de tiempos de reacción durante el cual la temperatura se mantuvo constante en $44^{\circ} \mathrm{C}$, es decir $\mathrm{T}_{\text {ref }}=44^{\circ} \mathrm{C}$. El uso de la expresión (4), evita los inconvenientes derivados de la fuerte correlación entre los factores preexponenciales y $E_{a}$.

\section{RESULTADOS DE LA REGRESIÓN DE LOS DATOS EXPERIMENTALES.}

\section{Estimación de las energías de activación de los coeficientes cinéticos}

Como fuera detallado en la sección 3, las distintas actividades entre los ensayos enmascaran la incidencia de la temperatura sobre los parámetros cinéticos. Para impedir este efecto, se modificó el nivel de temperatura durante el desarrollo de cada ensayo. De esta forma es posible determinar las energías de activación independientemente de las actividades propias de los ensayos. Todos los ensayos presentan en el inicio de la reacción un hidrocarburo insaturado con una concentración inicial alta, saturando la superficie catalítica y consumiéndose en orden cero. En estas condiciones los parámetros que se ponen de manifiesto son sólo los coeficientes cinéticos $k_{i}$ de la Tabla III. Los valores óptimos de las energías de activación se informan en la Tabla IV.

Tabla IV. Valores óptimos de energías de activación, Ea [cal/mol]. 


\begin{tabular}{|c|c|c|c|}
\hline $\mathrm{E}_{\mathrm{a} 1}$ & $(9.51 \pm 0.33) 10^{3}$ & $\mathrm{E}_{\mathrm{a} 5}$ & $(9.36 \pm 1.10) 10^{3}$ \\
\hline $\mathrm{E}_{\mathrm{a} 2}$ & $(11.29 \pm 0.98) 10^{3}$ & $\mathrm{E}_{\mathrm{a} 7}$ & $(11.07 \pm 0.60) 10^{3}$ \\
\hline $\mathrm{E}_{\mathrm{a} 3}$ & $(9.47 \pm 0.38) 10^{3}$ & $\mathrm{E}_{\mathrm{a} 8}$ & $(10.80 \pm 0.63) 10^{3}$ \\
\hline $\mathrm{E}_{\mathrm{a} 4}$ & $(6.70 \pm 0.82) 10^{3}$ & $\mathrm{E}_{\mathrm{a} 9}$ & $(4.13 \pm 1.44) 10^{3}$ \\
\hline
\end{tabular}

Las energías de activación $E_{\mathrm{a} 4}^{\mathrm{II}}, \mathrm{E}_{\mathrm{a} 6}$ y $\mathrm{E}_{\mathrm{a} 4}^{\mathrm{I}}$ pueden obtenerse a partir de sus relaciones de dependencia, ecs. 1 y 2 . Puede observarse que los valores modales de las energías de activación presentan, en general, una buena confiabilidad estadística. El intervalo de confianza relativamente más alto en la estimación de $\mathrm{E}_{\mathrm{a} 9}$ puede asignarse a su mismo valor bajo, cuyo efecto es una escasa influencia de la temperatura sobre la velocidad de la reacción en cuestión. El error relativo entre las estimaciones del modelo y los resultados experimentales resultó satisfactorio, resultando $\varepsilon=2.71 \%$.

Los valores de las energía de activación son comparables a los indicados por [7], a excepción de la correspondiente a la isomerización cBE-tBE que no es analizada por los autores.

\section{Estimación de la constante de adsorción de la especie reactiva del BY}

Para lograr la discriminación del parámetro cinético $K_{B Y}$ se hace necesario recurrir a ensayos como el de la Figura 4, que contienen desde el inicio BD y BY compitiendo entre si.

Debido a la estructura de la expresión cinética que se emplea, solo es posible el ajuste del valor relativo de la constante, $\left(\mathrm{K}_{\mathrm{BY}} / \mathrm{K}_{1 \mathrm{BE}}\right)$, ([5] y [12]).

A efectos de realizar la regresión de estos ensayos se emplean valores de parámetros cinéticos, que se mantienen fijos durante la regresión, obtenidos previamente a $44^{\circ} \mathrm{C}$ en [5] y [9]. Una base única de actividad del catalizador es necesario establecer, dado los problemas de desactivación que presentaron los ensayos, de forma de poder vincular los valores de los parámetros cinéticos. La zona donde sólo ocurren las reacciones de las olefinas está presente en todos los ensayos en [5] y [9] por lo que nos servirá de referencia para la vinculación. Un parámetro adicional, el factor de actividad de cada ensayo, afectando a los coeficientes cinéticos expresiones cinéticas, permite el empleo de los parámetros cinéticos estimados a partir de las distintas bases de datos.

El valor óptimo del parámetro cinético encontrado es $\left(\mathrm{K}_{\mathrm{BY}} / \mathrm{K}_{1 \mathrm{BE}}\right)=2050.77 \pm 276.38 \mathrm{a}$ $\mathrm{T}=44^{\circ} \mathrm{C}$. Los valores de composición predichos por el modelo ajustan adecuadamente los valores de composición experimentales, resultando el error relativo global, $\varepsilon=5.1 \%$.

\section{CONCLUSIONES}

Se estudió la hidrogenación selectiva, en fase líquida, de BY y BD en presencia de nbutenos sobre un catalizador comercial de $\mathrm{Pd} / \mathrm{Al}_{2} \mathrm{O}_{3}$ del tipo "cáscara de huevo". El estudio experimental se realizó en un reactor que opera en forma discontinua. El rango de concentraciones iniciales fue: $\mathrm{BD}, 1 \mathrm{BE}, \mathrm{tBE}$ y cBE hasta $10 \%, \mathrm{pH}_{2}$ entre 1.5 y $3.7 \mathrm{~atm}$ y cuatro niveles térmicos de temperaturas entre 25 y $65^{\circ} \mathrm{C}$.

A los fines de computar los valores intrínsecos de los parámetros cinéticos se incluyó, en el modelo empleado en la regresión, la resolución numérica de los balances de materia en el interior de la pastilla catalítica.

Sobre la base experimental recabada en el intervalo $25-65{ }^{\circ} \mathrm{C}$ se estimaron los valores óptimos de las energías de activación. Como resultado se consiguió una representación satisfactoria del conjunto de observaciones experimentales, con un error relativo de estimación, $\varepsilon=2.71 \%$. 
Se realizó una comparación de los valores propios de energías de activación con los informados en la bibliografía disponible. Los valores obtenidos en este estudio cinético y los reportados en las fuentes consultadas son similares.

Un análisis ulterior, incluyendo a la totalidad de la base de datos de trabajos previos ([5] y [9]), permitió la vinculación entre los distintos parámetros cinéticos a través de un factor de actividad, y la estimación de la constante de adsorción de la especie activa del BY, $\mathrm{K}_{\mathrm{BY}} / \mathrm{K}_{1 \mathrm{BE}}$. El ajuste permitió reproducir el conjunto de datos experimentales con un error relativo de estimación, $\varepsilon=5.1 \%$.

\section{Referencias bibliográficas}

[1] Derrien, M. L, Stud. Surf. Sci. Catal., 27, Cap.18, L. Cervený (editor), Elsevier, Amsterdam (1986).

[2] Bond, G. C., Webb, G., Wells, P. B. y Winterbottom, J. M., J. Catalysis; 1 (1962) 74-84.

[3] Boitiaux, J. P., Cosyns, J. y Robert, E., Appl. Catal., 35 (1987) 193-209.

[4] Molnár, A., Sárkány, A. y Varga M., J. of Mol. Catal. A: Chemical, 173, 185-221 (2001).

[5] Alves, J. A. Tesis Doctoral, Universidad Nacional de La Plata (2009).

[6] Schäfer, P., N.Wuchter, J.Gaube, Stud. Surf. Sci. Catal., 130 (2000) 2051-2056.

[7] Boitiaux, J.P., J.Cosyns, M.Derrien y G.Leger, AIChE Spring National Meeting, Trabajo 1453, Houston-USA (1985b).

[8] Alves J. A., Bressa S. P., Martínez O. M. y Barreto Guillermo F. Chem. Eng. J. 125 (2007) 131-138.

[9] Alves, J.A., Bressa, S.P., Martínez, O.M., Barreto, G.F., Chemical Engineering Research and Design (2010), doi:10.1016/j.cherd.2010.09.003.

[10] Ardiaca, N.O., Bressa S.P., Alves J.A., Martínez O.M., Barreto G.F, Catal. Today, 64 (2001) 205-215.

[11] Ardiaca N.O., S.P. Bressa, J.A. Alves, O.M. Martinez y G.F. Barreto, Stud. Surf. Sci. Catal., 133 (2001) 527-534.

[12] Ardiaca, N.O., Tesis Doctoral, Universidad Nacional de La Plata, (2002).

[13] Bressa S. P., Alves J. A., Martínez O. M. and Barreto Guillermo F., Chemical Engineering and Technology, 26, No 7, 783-789 (2003).

[14] Goetz, J., Yu Murzin, D. y Touroude, R., Ind. Eng. Chem. Res., 35 (1996) 703-711.

[15] Boitiaux, J. P., Cosyns, J. y Robert, E., Appl. Catal., 35 (1987) 193-209.

[16] Hub, S. y Touroude, R., J. of Catalysis, 114 (1988) 411-421.

[17] Stewart W. E., Caracotsios M. and Sørensen J. P. ; AIChE J., 38 ( 1992 ) 641.

[18] Bressa S.P., Mariani N. J., Ardiaca N. O., G. D. Mazza, O. M. Martínez y G. F. Barreto, Comp. Chem. Eng., 25 (2001) 1185-1198.

\section{Agradecimientos}

Los autores agradecen a la Universidad Nacional de La Plata - Argentina (Proyecto 11/I136), a la ANPCyT - Argentina (PICT'05 - 14/38336) y al CONICET (PIP 0304) por el apoyo financiero para la realización de este trabajo. J.A.A., O.M.M. y G.F.B. son miembros del CONICET y S.P.B. es miembro de la CIC-PBA. 